\title{
LINE-EDGE EXTRACTION BASED ON E-SPLINE ACQUISITION MODEL AND A FAST OPTIMIZATION ALGORITHM
}

\author{
Akira Hirabayashi ${ }^{1}$ and Pier-Luigi Dragotti ${ }^{2}$ \\ ${ }^{1}$ Dept. of Information Science and Engineering, Yamaguchi University \\ 2-16-1, Tokiwadai, Ube 755-8611, Japan \\ 2 Dept. of Electrical and Electronic Engineering, Imperial College London \\ Exhibition Road, London SW7-2AZ, United Kingdom
}

\begin{abstract}
We propose a line-edge extraction algorithm that uses an Espline data acquisition model and a fast optimization algorithm. The proposed method can retrieve line-edge parameters, including orientation, offset, and amplitude at sub-pixel accuracy almost independently of the resolution of the images. Because of the optimization approach, the proposed method is robust against model mismatch such as noise, point spread function (PSF) model mismatch, or step line-edge assumption. These properties are verified by simulations using images taken with a digital SLR camera.
\end{abstract}

Index Terms - Edge detection, line-edge, Hough transform, E-spline, optimization

\section{INTRODUCTION}

Straight line-edge is one of the most important image feature used in many applications including registration or vehicle navigation. The standard method to extract straight lines is the Hough transform and its extensions $[1,2]$. Such techniques, however, have limitations including the fact that many parameters need to be adjusted or the fact that they require high computational costs. Further, the Hough transform uses center position of a detected pixel as location of the line. Since this is not true generally, preciseness of the method degrades as the resolution of image decreases.

This difficulty was reduced by using more precise acquisition model. This approach was first taken by Baboulaz et al., who used B-spline functions for the acquisition model and proposed a method which can retrieve line-edge parameters including orientation, offset, and amplitude [3]. Hirabayashi et al. then used a trigonometric E-spline [4] for the model to improve noise robustness [5]. These methods can theoretically achieve infinite resolution. The key notion in the methods is a quantity computed by the product-sum of (horizontally or vertically) differentiated samples of image pixels and

This work was partially supported by KAKENHI, Grant-in-Aid for Scientific Research (C), 23500212, 2011. appropriate coefficients determined by the acquisition model. Its theoretical closed form provides analytic solutions for the line-edge parameters. If model mismatch is not too severe, then these analytic solutions can provide good estimates for the parameters. If the model mismatch becomes greater, however, further processing is necessary.

Hence, in this paper, we introduce an optimization approach to the acquisition model based approach. Our method performs line-edge extraction within a local area surrounding a pixel on which an edge is detected by a standard method like Canny. We define a criterion that evaluates closeness between the product-sum quantity computed from the pixels within the local area and that from the theoretical closed-form with candidate parameters. The criterion combines horizontal and vertical evaluations in a single formulation. Minimizers for the criterion are used to estimate the three parameters. Since the criterion is quadratic in terms of the amplitude, its analytic solution is available for fixed orientation and offset. Hence, the optimization is performed only over these two parameters. This problem is not globally convex, but locally around the optimum solution. Hence, we apply a gradient method with the analytic solutions for the initial values. Despite its computational simplicity the proposed approach provides extremely stable results as verified by simulations with real images obtained by a commercial digital SLR camera.

This paper is organized as follows. Section 2 provides mathematical preliminaries about the E-spline functions. In Section 3, the criterion that evaluates the aforementioned closeness is defined based on the closed forms for the horizontal and vertical product-sum quantities. We then propose our optimization approach. Section 4 is reserved for simulations. Section 5 concludes the paper.

\section{E-SPLINE FUNCTIONS}

Let $\vec{\alpha}=\left(\alpha_{0}, \alpha_{1}, \ldots, \alpha_{P}\right)$ be a $P+1$ dimensional complex vector and $\beta_{\alpha_{p}}(t)$ be

$$
\beta_{\alpha_{p}}(t)= \begin{cases}e^{\alpha_{p} t} & (-0.5 \leq t<0.5), \\ 0 & (t<-0.5, t \geq 0.5) .\end{cases}
$$




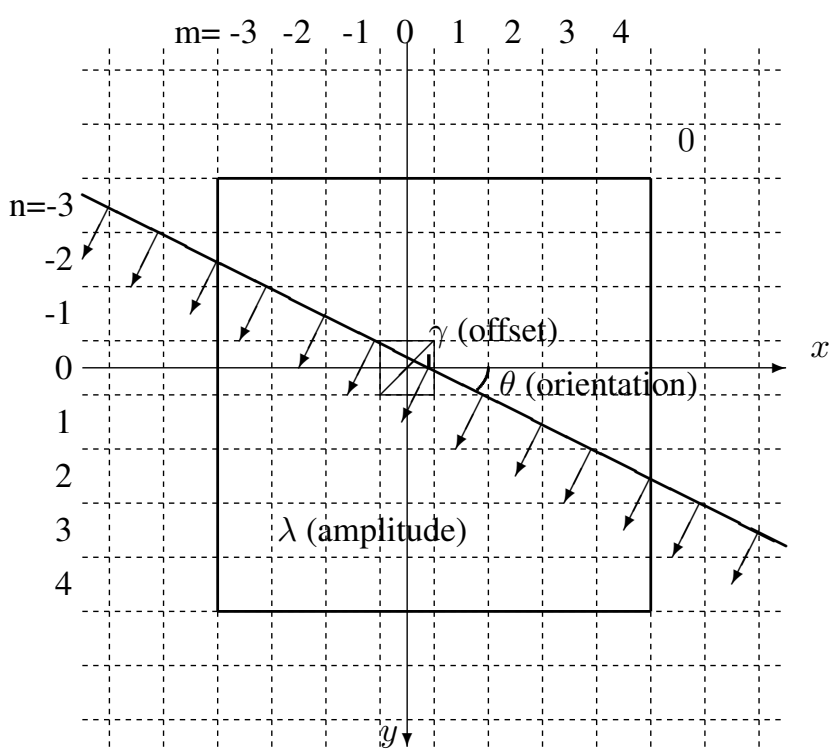

Fig. 1. Description parameters for a step line-edge. The $x y$ coordinates are local ones whose origin is the center of the pixel detected as an edge. The grid shows sampled pixels.

Let $\beta_{\vec{\alpha}}(t)$ be the convolution of the $P+1$ functions $\beta_{\alpha_{p}}(t)$ as

$$
\beta_{\vec{\alpha}}(t)=\left(\beta_{\alpha_{0}} * \beta_{\alpha_{1}} * \ldots * \beta_{\alpha_{P}}\right)(t) .
$$

The function $\beta_{\vec{\alpha}}(t)$ is called the E-spline of order $P$ and proper linear combination of shifted versions of this spline can reproduce exponentials of the form $e^{\alpha_{p} t}, p=0, \ldots, P[4]$.

\section{STEP LINE-EDGE EXTRACTION}

By using orientation $\theta$, offset $\gamma$, and amplitude $\lambda$, which are defined as in Fig. 1, a step line-edge can be expressed as

$$
f(x, y)=\lambda H(-x \sin \theta+y \cos \theta+\gamma \sin \theta),
$$

where $H(t)$ is the unit step function whose value is 1 if $t \geq 0$ and 0 if $t<0$. This continuous expression of the step edge is sampled by the integer-shifted version of a sampling kernel $\psi(x) \psi(y)$ as

$$
g[m, n]=\langle f(x, y), \psi(x-m) \psi(y-n)\rangle+\epsilon[m, n],
$$

where $\epsilon[m, n]$ is additive noise. The sampling kernel $\psi(t)$ is modeled by the trigonometric E-spline of the first order $(P=$ $1)$, which is the one where $\vec{\alpha}=\left(i \omega_{0},-i \omega_{0}\right)$ with $\omega_{0} \in \mathbb{R}$, and is given by

$$
\beta_{\vec{\alpha}}(t)=\left\{\begin{array}{cl}
\sin \omega_{0}(t+1) / \omega_{0} & (-1 \leq t<0), \\
-\sin \omega_{0}(t-1) / \omega_{0} & (0 \leq t<1), \\
0 & (t \leq-1, t>1) .
\end{array}\right.
$$

When $\omega_{0}$ tends to zero, the trigonometric E-spline converges to the B-spline of the first order.
The main stream of the algorithm we propose here is similar to those in [3] or [5]. First, edge pixels are detected by a conventional method like Canny operator. Then, for each pixel detected as an edge, the surrounding pixel area is extracted, and the three parameters are computed from the pixels in the area. To suppress extraction errors, similar edges are merged, while other edges are discarded. Within these steps, we mainly discuss the second one. Therefore, the indices $m$ and $n$ are assigned in a local manner: the focused detected pixel is set to $m=n=0$. The local area size is chosen as $8 \times 8$ pixels since those affected by the focused edge are mostly within this area. This is because the sampling kernel is modeled by the E-spline of the first order (its support width is two). Hence, the indices $m$ and $n$ are from -3 to 4 (see Fig. 1).

To retrieve the parameters $\theta, \gamma$, and $\lambda$ from the pixel values $g[m, n]$, we first compute a horizontal differentiated sample $d_{H}[m, n]$ which is given by $g[m+1, n]-g[m, n]$. We then compute product-sum of $d_{H}[m, n]$ and coefficients $C_{m}^{\left(\alpha_{p}\right)}$ :

$$
\tau_{n, p}^{(H)}=\sum_{m=-3}^{3} C_{m}^{\left(\alpha_{p}\right)} d_{H}[m, n] .
$$

The coefficients $C_{m}^{\left(\alpha_{p}\right)}$ are determined so that they satisfy

$$
\sum_{m=-\infty}^{\infty} C_{m}^{\left(\alpha_{p}\right)}\left(\beta_{\alpha_{2}} * \psi\right)(t-m)=e^{\alpha_{p} t}
$$

for $p=0,1,2$, where $\beta_{\alpha_{2}}(t)$ is defined by (1) with $\alpha_{2}=0$, and they can be computed by

$$
C_{m}^{\left(\alpha_{p}\right)}=e^{m \alpha_{p}} /\left\{\sum_{k=-P^{\prime}}^{P^{\prime}} e^{k \alpha_{p}}\left(\beta_{\alpha_{2}} * \psi\right)(-k)\right\},
$$

where $P^{\prime}$ is the maximum integer not exceeding $(P+2) / 2$. Note that the convolved sampling kernel $\left(\beta_{\alpha_{2}} * \psi\right)(t)$ can produce $e^{\alpha_{p} t}$ for $p=0,1,2$.

To show a closed form of $\tau_{n, p}^{(H)}$, let us define

$$
\mu_{n, p}^{(H)}(\theta, \gamma)=-\operatorname{sgn}(\sin \theta) e^{\alpha_{p}\left(\gamma+\frac{n}{\tan \theta}-\frac{1}{2}\right)} \Psi\left(\frac{\alpha_{p}}{\tan \theta}\right),
$$

where $\operatorname{sgn}(t)$ is the function whose value is 1 if $t>0,0$ if $t=0$, and -1 if $t<0$ and $\Psi(s)=\int_{-\infty}^{\infty} \psi(t) e^{s t} d t$. Assume that $d_{H}[m, n]$ is equal to zero for $|m| \geq 4$. Then, as shown in [5], it holds for $p=0,1,2$ that

$$
\tau_{n, p}^{(H)}=\lambda \mu_{n, p}^{(H)}(\theta, \gamma)
$$

This equation yields closed formulas for $\tan \theta, \gamma$, and $\lambda$ [5]. If model mismatch is not too severe, then $\tau_{n, p}^{(H)}$ can be computed by (3) and the closed formulas can provide good estimates for $\lambda, \theta$, and $\gamma$. However, if model mismatch cannot be ignored, it is getting hard for the formulas to work precisely. To overcome this limitation, we search for $\theta, \gamma$, and $\lambda$ by which the 
Standard Dev. [dB]

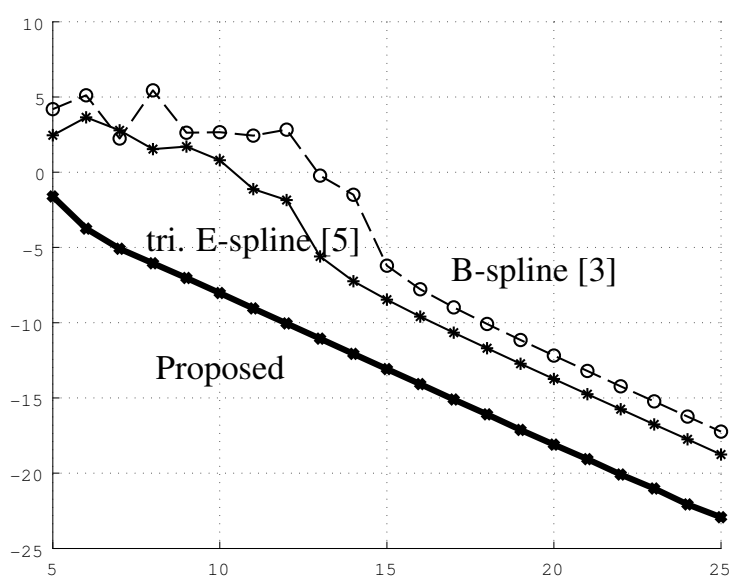

(a) $\tan \theta$

Input SNR [dB]
Standard Dev. $[\mathrm{dB}]$

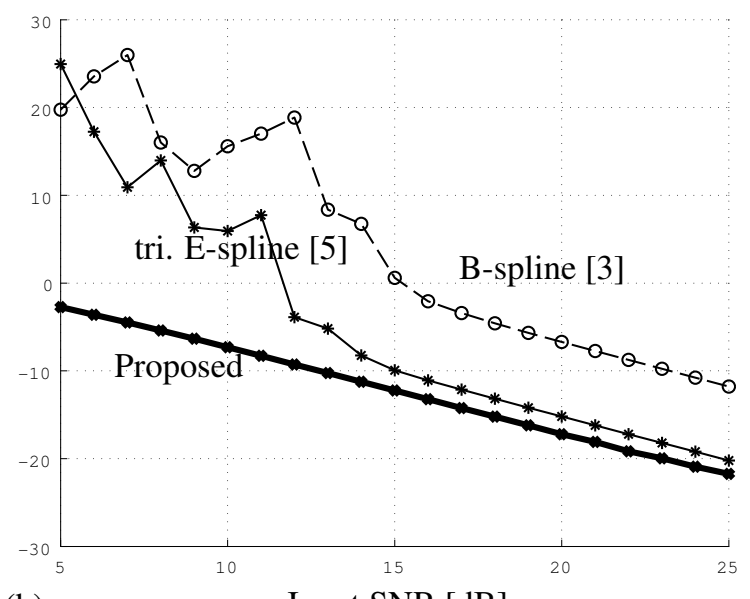

(b) $\gamma$

Input SNR [dB]

Fig. 2. Standard deviations $[\mathrm{dB}]$ of estimated results for (a) $\tan \theta$ and (b) $\gamma$ in terms of signal-noise ratio from $5[\mathrm{~dB}]$ to $25[\mathrm{~dB}$ ].

right-hand side in (6) best approximates the left-hand side for all $n=-1,0,1$ and $p=0,1,2$.

As well as the horizontal one, we can do the same processing vertically. That is, let us compute differentiated samples vertically as $d_{V}[m, n]=g[m, n+1]-g[m, n]$. Then, the product-sum of $d_{V}[m, n]$ and coefficients $C_{n}^{\left(\alpha_{p}\right)}$ is computed by

$$
\tau_{m, p}^{(V)}=\sum_{n=-\infty}^{\infty} C_{n}^{\left(\alpha_{p}\right)} d_{V}[m, n]
$$

and $\tau_{m, p}^{(V)}$ has the following closed form:

$$
\tau_{m, p}^{(V)}=\lambda \mu_{m, p}^{(V)}(\theta, \gamma),
$$

where

$$
\mu_{m, p}^{(V)}(\theta, \gamma)=\operatorname{sgn}(\cos \theta) e^{\alpha_{p}\left\{-(\gamma-m) \tan \theta-\frac{1}{2}\right\}} \Psi\left(\alpha_{p} \tan \theta\right) .
$$

Proof. The vertically differentiated sample $d_{V}[m, n]$ can be expressed as

$d_{V}[m, n]=\left\langle\frac{\partial f(x, y)}{\partial y}, \psi(x-m)\left(\beta_{P+1} * \psi\right)(y-n-0.5)\right\rangle$.

It follows from (2) that

$$
\frac{\partial f(x, y)}{\partial y}=\lambda \delta(-x \sin \theta+y \cos \theta+\gamma \sin \theta) \cos \theta,
$$

where $\delta(t)$ is Dirac's delta function. Substituting these relations and (4) into (7) yields

$$
\tau_{m, p}^{(V)}=\lambda e^{\alpha_{p}\{-(\gamma-m) \tan \theta-0.5\}} \Psi\left(\alpha_{p} \tan \theta\right)
$$

if $\cos \theta>0$. If $\cos \theta<0$, then the right-hand side becomes its negative version. If $\cos \theta=0$, then $\tau_{m, p}^{(V)}=0$. These relations are combined into (8).
Let us define eighteen dimensional vectors $\boldsymbol{\tau}$ and $\boldsymbol{\mu}(\theta, \gamma)$ as

$$
\begin{gathered}
\boldsymbol{\tau}=\left(\tau_{-1,0}^{(H)}, \tau_{-1,1}^{(H)}, \tau_{-1,2}^{(H)}, \tau_{0,0}^{(H)}, \ldots \tau_{1,1}^{(V)}, \tau_{1,2}^{(V)}\right)^{T}, \\
\boldsymbol{\mu}(\theta, \gamma)=\left(\mu_{-1,0}^{(H)}(\theta, \gamma), \mu_{-1,1}^{(H)}(\theta, \gamma), \mu_{-1,2}^{(H)}(\theta, \gamma),\right. \\
\left.\mu_{0,0}^{(H)}(\theta, \gamma), \ldots, \mu_{1,1}^{(V)}(\theta, \gamma), \mu_{1,2}^{(V)}(\theta, \gamma)\right)^{T} .
\end{gathered}
$$

Then, the differences between the left and right hand sides in (6) and (8) can be simultaneously evaluated by

$$
J_{o}(\theta, \gamma, \lambda)=\|\lambda \boldsymbol{\mu}(\theta, \gamma)-\boldsymbol{\tau}\|^{2} .
$$

For fixed $\theta$ and $\gamma$, the optimal $\lambda$ is obviously given by

$$
\lambda_{\text {opt }}(\theta, \gamma)=\left\langle\boldsymbol{\tau}, \frac{\boldsymbol{\mu}(\theta, \gamma)}{\|\boldsymbol{\mu}(\theta, \gamma)\|^{2}}\right\rangle .
$$

Hence, $\theta, \gamma$, and $\lambda$ which minimize $J_{o}$ are given by $\theta$ and $\gamma$ which minimize

$$
J(\theta, \gamma)=J_{o}\left(\theta, \gamma, \lambda_{\mathrm{opt}}(\theta, \gamma)\right),
$$

and then $\lambda_{\text {opt }}(\theta, \gamma)$ with the resultant values. Note that the proposed approach elegantly combines horizontal and vertical evaluations into a single formulation in the criterion $J$.

Since line-edge in our formulation has 'direction', a horizontal edge, for example, with 0 and $\lambda$ for the top and bottom, respectively, is different from that with the counter order. Hence, we have to search for $\theta$ from $-\pi$ to $\pi$ rather than $-\pi / 2$ to $\pi / 2$. On the other hand, since the edge should exist near the focused pixel, $\gamma$ is supposed to be a small number. Hence, we searched for $\gamma$ in the range of -1.5 to 1.5. The problem of finding the minimizer within this area is not globally convex, but we can treat it convex around the optimum values. Therefore, we used a gradient method with the analytic solutions for the initial values. 


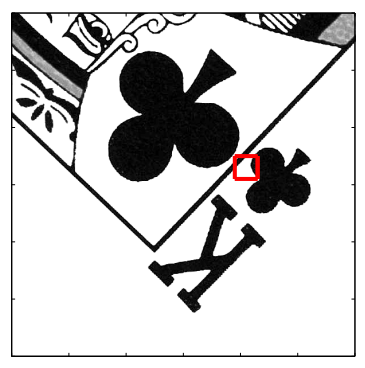

(a)

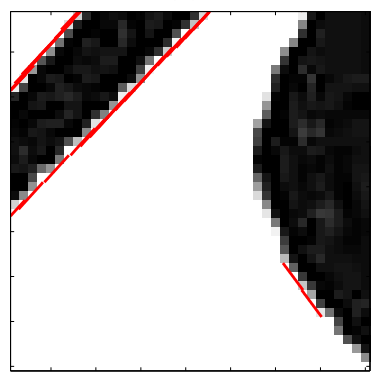

(c)

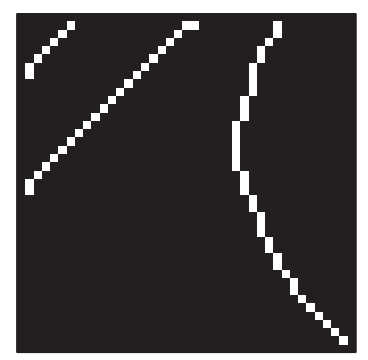

(b)

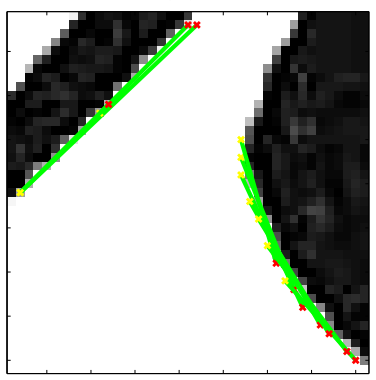

(d)
Fig. 3. Edge extraction results by the proposed algorithm: (a) original image, (b) Canny edge detection results, (c) results by the proposed method, (d) results by the Hough transform.

\section{SIMULATIONS}

\subsection{Resilience to Noise}

To evaluate noise precisely, we applied the proposed method and the closed form approaches to a synthesized image with noise. The noiseless image is a step line-edge with $\theta=-\pi / 4$, $\gamma=0$, and $\lambda=100$. The noise $\epsilon[m, n]$ is independently and identically generated from Gaussian distribution with standard deviation $\sigma$, which was determined so that the signalto-noise ratios (SNRs), defined by $10 \log _{10}(\lambda / \sigma)$, were from $5 \mathrm{~dB}$ to $25 \mathrm{~dB}$ with step size $1 \mathrm{~dB}$. We used $\omega_{0}=\pi / 8$.

The results are shown in Fig. 2, in which (a) and (b) indicate the standard deviations of estimated values for $\tan \theta$ and $\gamma$, respectively. They are shown in $\mathrm{dB}$ in order to clearly show the difference for the large SNR values. In each figure, the thick and thin solid lines show the results obtained by the proposed method and the closed form approach based on Espline, respectively. The dashed line shows the result by the B-spline counterpart. We can see that the proposed method constantly outperforms both the closed-form approaches. The gains of the proposed method compared to the closed form approach with E-spline are at least $4.0[\mathrm{~dB}]$ and $1.5[\mathrm{~dB}]$ for $\tan \theta$ and $\gamma$, respectively. It should be noted that the proposed method performs very stably even when the input SNR is less than $11[\mathrm{~dB}]$. These results show the robustness against noise of the proposed approach.

\subsection{Real Images}

We applied the proposed method to the real image obtained by a Nikon D50 SLR camera. Its PSF is simply approximated by the trigonometric E-spline of the first order with $\omega_{0}=\pi / 8$ without any calibration. Fig. 3 (a) shows the original image, Fig. 3 (b) shows the Canny edge detection results. Figs. 3 (c) and (d) show the extracted edges for the small area in the box indicated in Fig. (a) by the proposed method and the Hough transform, respectively. We can see that the Hough transform extracts many wrong straight line edges along the curve on the right while the proposed method does more precise results and less wrong ones. We also note that the Hough transform could not extract the top left straight line edge because the area shown in Fig. (d) was not sufficient. Even though the PSF for the camera is unknown, the proposed method showed the good performance. This means that the proposed approach is robust against the PSF model mismatch. The computational time for the simulation by the proposed method was 0.7[s] which can be accelerated by more dexterous initial values.

\section{CONCLUSION}

We proposed a line-edge extraction method using an E-spline acquisition model and a fast yet effective optimization algorithm. The proposed method estimated line-edge parameters by minimizing a criterion that evaluates closeness between quantities computed from theoretical closed form and pixel values. The proposed method is robust against model mismatch such as noise, PSF model, or step line-edge assumption as shown by simulations with synthetic and real images.

\section{REFERENCES}

[1] N. Aggarwal and W.C. Karl, "Line detection in images through regularized Hough transform," IEEE Trans. Image Processing, vol. 15, no. 3, pp. 582-591, 2006.

[2] D. Shi, L. Zheng, and J. Liu, "Advanced Hough transform using a multilayer fractional Fourier method," IEEE Trans. Image Processing, vol. 19, no. 6, pp. 1558 -1566, 2010.

[3] L. Baboulaz and P.L. Dragotti, "Exact feature extraction using finite rate of innovation principles with an application to image super-resolution," IEEE Trans. Image Processing, vol. 18, no. 2, pp. 281-298, 2009.

[4] M. Unser and T. Blu, "Cardinal exponential splines: Part I-Theory and filtering algorithms," IEEE Trans. Signal Processing, vol. 53, no. 4, pp. 1425-1438, 2005.

[5] A. Hirabayashi and P.L. Dragotti, "E-spline sampling for precise and robust line-edge extraction," in Proc. International Conference on Image Processing (ICIP 2010), Hong Kong, 2010, pp. 909-912. 